\section{Editorial}

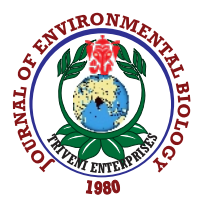

DOI : http://doi.org/10.22438/jeb/41/4/Editorial

\title{
Wonderful world of microbes: the JEB way
}

Life under a microscope is fascinating. The role of microbes in maintaining the diversity of life as well as ecological balance has been a special area of interest of the researchers. Role of microbes in biogeochemical process have been phenomenal. However, in spite of their plethora of activities, the mechanisms underlying their diversified role are not charted out clearly.

Journal of Environmental Biology plays a pivotal role in highlighting the world of microbes and this issue deftly deals with the role of bacteria in delignifying the golden fibre which is of immense commercial importance. The bacterial diversity in the rough terrain of Ladakh along with the fatty acid profile of Antarctic cyanobacteria are the brimming bowls of information of scientific interest. The bacterial pathogens that have been the cause of woes in case of cow heifers of Himalayan region, have been vividly depicted in one of the

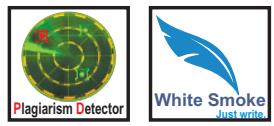
treatises and it opens the floodgate of caution regarding the risk factor of the disease. The wonder fungus Trichoderma, has been instrumental in management of soil borne disease as well as in induction of systematic acquired resistance in several crops of commercial importance. Its role in removal of toxic heavy metal ions had been accoladed globally and the way a time honoured technology of protoplast fusion has been deployed to get a zinc tolerant fungi is really commendable. Finally, bacterial importance in human medical science can never be undermined and the bacterial composition in the subgingival plaque of women with peridontitis has been aptly dealt with in one of the contributions.

Microbes, being ubiquitous in nature, a considerable interest is generated regarding the microbial community, which may be structured by a large number of factors including environment. The environment is supposed to select and sort microbes according to their ecological niche. The response of microbes to environmental conditions and changes thereof is mediated by a complex combination of adjustment, replacement, and species interaction mechanisms, all facilitated by their fast population growth rates. Advances in molecular biology have enabled the academia, to understand the paradigm of microbial diversity in a new perspective. However, the challenge in microbial ecology which persists is whether the microbes show unique features or have pattern in common with macroorganism. As, the process involved in structuring the respective communities are different from each other, if is highly unlikely, that response follows the same patterns among group of microorganisms.

JEB has been the corner stone of divulging multitude of information regarding an amalgamation of microbes and the environment and is destined to continue its journey in the same path. 